\title{
LA DIGNIDAD HUMANA Y LA ÉTICA MÉDICA EN ESLOVAQUIA ${ }^{32}$
}

\author{
Human Dignity and the Medical Ethics in Slovakia
}

Vasil Gluchman

(UniPo, Eslovaquia)

\begin{abstract}
Resumen
La dignidad humana es un concepto significativo en las Declaraciones de la UNESCO sobre bioética, así como también en la ética médica (incluyendo a todas las profesiones médicas). En Eslovaquia, los primeros intentos más significativos de formalizar éticas profesionales ocurrieron durante la segunda mitad de la década de 1990. Tradicionalmente, la esfera de la biomedicina (la ética profesional de los doctores, enfermeras, etc.) tuvo el desarrollo más intenso entre las éticas profesionales en la República Eslovaca. Sin embargo, los temas de ética médica son muy a menudo reducidos sólo al código de ética. Me gustaría investigar la idea de dignidad humana en las relaciones entre doctores, enfermeras y pacientes en Eslovaquia. La dignidad humana es un concepto que usamos para describir un agregado de valores y cualidades de alguien o algo que merece estima y respeto. Sin embargo, ¿cómo es la dignidad humana de los doctores, enfermeras y pacientes? ¿Es un valor primario de todos los seres humanos sin diferencias en su estatus social o depende de habilidades de agentes morales (incluyendo a los doctores, enfermeras y pacientes)? ¿Cómo es preservada la dignidad humana de doctores, enfermeras, otros trabajadores de la salud y pacientes por los códigos de ética de las profesiones médicas en Eslovaquia?
\end{abstract}

Palabras clave: Dignidad humana | Eslovaquia | Doctores | Enfermeras | Trabajadores de la Salud.

\begin{abstract}
Human dignity is a significant concept of the UNESCO Declarations on bioethics as well as medical ethics (including all medical professions). In Slovakia, the first more significant attempts in development of professional ethics occur in the second half of the 1990s. Traditionally, the sphere of biomedicine (professional ethics of doctors, nurses, etc.) is the most intensive development of professional ethics in Slovakia. However, the issues of medical ethics are very often reduced only to the ethical code. I would like to research idea of human dignity in relations among doctors, nurses and patients in Slovakia. Human dignity is a concept that we use to describe an aggregate of values and qualities of someone or something that deserve esteem and respect. However, what is human dignity of doctors, nurses and patients? Is it a primary value of all human beings without differences in their social status or it depends on abilities of moral agents (including doctors, nurses and patients)? How is kept human dignity of doctors, nurses, health care workers and patients by ethical codes of medical professions in Slovakia at all?
\end{abstract}

Keywords: human dignity, Slovakia, doctors, nurses, health care workers

32 Este artículo, y el proyecto en el que está enmarcado, está sostenido por el subsidio VEGA 1/0629/15 Ethics of social consequences in context of contemporary ethical theories. 


\section{Introducción}

En el Artículo 1 de la Declaración Universal sobre Genoma Humano y Derechos Humanos (1997), se afirma que "el genoma humano remarca la unidad esencial de todos los miembros de la familia humana, y reconoce su dignidad innata y diversidad. En un sentido simbólico, este es la herencia de la humanidad" (UNESCO, 2000, p. 3). En el Artículo 1 de la Declaración Universal sobre Bioética y Derechos Humanos (2005), se afirma aún más vagamente que "la dignidad humana, los derechos y la libertad esencial son totalmente respetados” (UNESCO, 2006, p. 6).

Recientemente, han comenzado a ser mencionadas más y más a menudo en los círculos bioéticos académicos, así como también en los médicos, algunas dudas con respecto a la necesidad o inutilidad del término "dignidad humana". Un número de autores señala la vaguedad de las definiciones existentes del término "dignidad", o "dignidad humana" (Cochrane, 2010; Foster, 2015; Macklin, 2003; Palacios-Gonzáles, 2015; Pilkington, 2016; Pinker, 2008; Pirhonen, 2015; Schüklenk-Pacholczyk, 2010). Ellos también llaman la atención sobre la redundancia, inutilidad e, incluso, nocividad de este término. Ruth Macklin sostiene que la dignidad humana no representa otra cosa que el respeto hacia las personas y a su autonomía. En su opinión, la conclusión es clara: la dignidad en la ética médica es un término innecesario y puede ser eliminado sin ninguna pérdida de significado (Macklin, 2003, p. 1420).

Con respecto a lo anterior, Alasdair Cochrane añade otro argumento, sosteniendo que el término "dignidad humana" es reaccionario, en tanto que crea obstáculos sin fundamento al progreso medicinal, causando grandes daños. En su artículo Undignified Bioethics, analiza cuatro concepciones de la dignidad: la dignidad como conducta virtuosa, la dignidad como valor interno, la comprensión kantiana de la dignidad y la dignidad como integridad de la especie. Cochrane admite que es posible hallar una interpretación coherente de la dignidad; sin embargo, rechaza la última defensa de la dignidad no sólo porque es poco clara, reaccionaria y sin sentido, sino porque principalmente está basada en argumentos éticos insostenibles. Según Cochrane, ninguna de estas concepciones de la dignidad es en conjunto aplicable en bioética a, por ejemplo, la resolución de problemas de ética médica, y especialmente de ética clínica. Él sostiene que la popularidad de un término ético particular no implica que estemos comprometidos a aceptarlo para siempre. Las tendencias en el discurso ético están cambiando y los especialistas en ética tienen que aceptar estos cambios, aunque sea a un nivel bajo. Si los términos son poco claros, es tarea de los expertos el clarificarlos. Si, sin embargo, los términos se prueban innecesarios, o incluso, inútiles, deben ser rechazados. De este modo, Cochrane se inclina hacia una bioética sin dignidad, hacia una "bioética indignificada" (Cochrane, 2010, pp. 234-241).

Steven Pinker presenta una opinión similar cuando señala la relatividad, intercambiabilidad y nocividad del uso del término "dignidad humana". En su opinión, la relatividad de este término descansa en el ser susceptible al tiempo, lugar y comprensión del receptor. Pinker sostiene que muchas veces se ha probado que la dignidad es un valor trivial, intercambiable con los de vida, salud y seguridad. La nocividad de este término se manifiesta a menudo en su defensa en la forma de rasgos despóticos de la conducta, tales como la fatwa contra Salman Rushdie, la caricaturas danesas de Mahoma, etc. Por otro lado, Pinker también es consciente del potencial significativo incluido en este término. Él sostiene que cada uno de nosotros quiere ser percibido con dignidad. Esto es uno de los intereses del hombre, tal como la integridad corporal o las pertenencias personales. La arriba mencionada percepción de la dignidad es significativa con respecto a la biomedicina, especialmente cuando concierne a la curación de los pacientes. Otra razón para aceptar a la dignidad es, según Pinker, la posibilidad de que su eliminación lleve a la pérdida de inhibiciones como la tortura, el tormento y la humillación de otras personas, tal como ocurrió, por ejemplo con el caso de los judíos en la Alemania nazi. Sin embargo, Pinker ve un problema en el hecho de que, en muchos casos, los bioeticistas religiosos conservadores (theocon bioethicists) consideran a la ciencia ficción como profecía, lo que les lleva a -en el nombre de la salvación de la dignidad - inhibir progresos en la investigación biomédica, que podrían ayudar a disminuir el sufrimiento de mucha gente enferma (Pinker, 2008, pp. 28-31). 
Sin lugar a dudas, la dignidad humana es un problema teórico interesante e importante, y, de la misma manera, es un problema ético. De modo que como problema moral práctico, su solución podría tener un impacto altamente significativo en los desarrollos futuros de la bioética, la ética médica, y la investigación biomédica, incluyendo la investigación en tecnología genética. Por lo tanto, intentaré formular mi propia posición, la que, por un lado, resulta de mi interpretación de la dignidad humana, y también propone respuestas a al menos algunas de las objeciones evocadas por los autores arriba mencionados, o en general hacia aquellos que critican el uso del término "dignidad humana" en bioética.

Ante todo, me gustaría señalar que, en la mayoría de los casos, los críticos operan con un modelo de interpretación de la dignidad humana estático o conservador, basado, hasta cierto punto, en enfoques deontológicos de la dignidad humana y la moralidad en general. Sobre todo entienden a la moralidad como un conjunto de valores estáticos, o estables, con el foco puesto en respetar principios morales existentes, reglas, normas y valores, mientras un rol mayor es jugado por el respeto y el cumplimiento de obligaciones. La interpretación de Cochrane de la dignidad humana también cabe en este ámbito; él la percibe como un cierto modelo de conducta que necesita ser cumplido, o un conjunto de ciertos valores estables y estáticos resultantes de la naturaleza humana. De carácter similar es la crítica de Pinker a la interpretación de la dignidad humana hecha por el American Council for Bioethics del período de la presidencia de George Bush, cuando las visiones cristianas conservadoras sobre la dignidad humana estaban dominadas por la opinión concerniente al don de la vida y al origen divino del hombre.

\section{El valor de la dignidad humana en la ética médica en Eslovaquia}

A diferencia de los críticos, no soy de la opinión de que el término "dignidad humana" pueda ser reemplazado por el de "respeto" hacia una persona y su autonomía, dado que esto reduce el significado del valor de la dignidad humana a una mera dimensión funcional de la existencia humana y esto, en un cierto grado, se acerca a lo que Cochrane criticó con respecto a la comprensión de la dignidad como una especie de integridad, existencia dignificada del hombre. El programa nazi de "la solución final al problema judío" estaba basado en el hecho de que los nazis "gracias a" las leyes racistas, privaron a los judíos del estatus de personas y de su autonomía, lo que, como resultado, facilitó todo el programa de exterminio incluyendo experimento inhumanos sobre prisioneros en campos de concentración (el ejemplo más conocido es el de Josef Mengele y sus experimentos en Auschwitz).

En lugar de un modelo de dignidad estático (conservador), o funcional, que es el tema de un número de puntos de vista y estipulaciones críticos justificados, estoy ofreciendo un modelo dinámico para interpretar a la dignidad humana basado en una intersección de los valores de humanidad [humaneness], dignidad humana, derecho moral (a la vida) y consecuencias (sociales) positivas (Dubiel-Zielińska, 2013, pp. 173-176; Gluchmanová, 2009, pp. 243-250; Kalajtzidis, 2013, pp. 159-170; Lešková Blahová, 2009, pp. 90-98).

Acepto que fundamentalmente la dignidad es un concepto que usamos para describir un agregado de valores y cualidades de alguien o algo que merece estima y respeto. El valor primario que crea el derecho a tener dignidad es la vida. El grado de dignidad de una forma de vida particular depende de su lugar en la escala evolutiva. Los seres humanos son la forma más alta de vida de modo que poseen el más alto grado de dignidad. Tal comprensión del valor de la dignidad, incluyendo la dignidad humana, representa en su definición básica una forma o manifestación de biocentrismo moral. Sin embargo, ciertamente no significa una devaluación del valor de la dignidad, incluyendo a la dignidad humana. Al contrario, aumenta su alcance, de modo que se extiende también más allá de la categoría de humanidad (Gluchman, 2012, pp. 20-25; Misseri, 2015, pp. 201-202; Sachdev, 2015, pp. 206-207).

En Eslovaquia, los primeros intentos más significativos en el desarrollo de éticas profesionales 
ocurren en la segunda mitad de la década de 1990. Tradicionalmente, la esfera de la biomedicina (éticas profesionales de doctores, enfermeras, etc.) tuvo el desarrollo más intenso de éticas profesionales en Eslovaquia. Sin embargo, los temas de la ética médica están muy a menudo reducidos sólo al código de ética (Komenská 2011, pp. 25-32; Komenská, 2012b, pp. 194-198). Aquí me gustaría investigar la idea de la dignidad humana en las relaciones entre doctores, enfermeras y pacientes en Eslovaquia. La dignidad humana es un concepto que podemos usar para describir un agregado de valores y cualidades de alguien o algo que merece estima y respeto. Sin embargo, ¿cuál es la dignidad humana de los doctores, las enfermeras y los pacientes? ¿Es este un valor primario de todos los seres humanos sin diferencias en su estatus social o depende de sus habilidades como agentes morales (incluyendo a los doctores, enfermeros y pacientes)? ¿Cómo se mantiene la dignidad humana de los doctores, las enfermeras, otros trabajadores de la salud y los pacientes con los códigos de ética de las profesiones médicas en Eslovaquia?

El análisis de los códigos de ética de los trabajadores de la salud eslovacos forma dos requisitos básicos para su interés actual. El primero está basado en el cambio de la ética médica al formular sus principios para enfocarse sobre el paciente, el receptor del cuidado sanitario. Esto remarca el rol del paciente y el respeto a la dignidad y autonomía en la toma de decisiones y el reparto del cuidado sanitario. Basado en el breve análisis presentado puede observarse que la ética profesional tiene a ser vista como siguiendo el modelo del sistema de atención sanitaria orientado al paciente y respetando su autonomía y valores individuales en Eslovaquia.

Katarína Komenská sostiene que en particular, las actividades de los trabajadores de la salud están formuladas como actividades "de acuerdo con los principios de humanidad, en un espíritu de respeto por la vida humana desde su comienzo hasta su fin respetando la dignidad de un ser humano" (Zákon č. 578/2004). El código de ética de los enfermeros ha añadido esta formulación cuando espitula que un trabajador sanitario "sopesa la vida humana, los derechos humanos y la dignidad de cada individuo como un ser humano único, aceptando al cliente-paciente en su situación de salud" (Zákon č. 311/2002). Las parteras están en el código de su profesión incluso obligadas por el requisito de apoyar el derecho de las pacientes a "participar activamente en las decisiones de su cuidado [...] el derecho a hablar por sí mismas en asuntos relacionados con su salud y la salud de sus familias" (Zákon č. 311/2002; Komenská, 2012a, p. 96).

Según Adela Lešková Blahová, es positivo que ambos códigos busquen declarar los presupuestos del valor clave en el campo de la atención sanitaria. Ambos afirman que la obligación del profesional de la salud es "preservar la vida, proteger y restaurar la salud, evitar la enfermedad, disminuir el sufrimiento", pero también ser independiente en sus decisiones profesionales, responsable y confiable, prestar atención a su desarrollo profesional continuo, actuar de acuerdo con los principios de humanidad, y respetar la dignidad de la vida humana, si es necesario. Prosiguiendo con las regulaciones legales vinculantes (Etický kódex zdravotníckeho pracovníka, 2004, p. 5752; Etický kódex, 2002, p. 2184), puende ser hallados valores universales en el primer artículo, titulado Obligaciones generales de un profesional de la salud (como doctores y dentistas), el que prácticamente corresponde al actual sistema sanitario en Eslovaquia (Etický kódex zdravotníckeho pracovníka, 2004). Deficiencias más serias, sin embargo, surgen con respecto a la declaración de valores espécificos de la profesión médica. Dado que es casi idéntica para el documento de ambas profesiones (como un doctor, así como también el sentido semánticamente más amplio de trabajador de la salud), es muy difícil identificar valores específicos para cada uno de ellos por separado (Lešková Blahová, 2012, p. 109).

Lešková Blahová afirma que los principios fundamentales éticos y profesionales de la profesión médica que podríamos considerar son (tomados del texto de ambos documentos): profesionalismo, legalidad, prohibición del abuso de poder, rendición de cuentas, equidad e imparcialidad, cortesía, apertura en la comunicación, colaboración colegiada (multidisciplinaria), protección de los datos personales, respeto por la autonomía de los pacientes, acción humana, dignidad humana y respeto por los otros como fue afirmado arriba. El inconveniente es que los estándares y las conductas éticos y profesionales esperados no los hallamos concentrados en un solo lugar, o en un solo artículo, sino "entre líneas" a través de todo el documento (Lešková Blahová, 2012, pp. 109-110). 
En su opinión, en un examen más profundo del artículo Atención sanitaria profesional y ejercicio de su profesión (como médicos y dentistas) hallamos que se aspira a presentar algunas situaciones posibles de conflicto moral en el ejercicio de una profesión sanitaria (aunque no reducida sólo a ellas) (Etický kódex zdravotníckeho pracovníka, 2004). Podemos hallar, por ejemplo, la obligación a desenvolverse en tareas profesionales "[...] incluso en situaciones de amenaza pública y de desastres naturales o de otra naturaleza" o "[...] prescribir y administrar drogas, que implican adicción, o aquellas que tienen efectos de dopaje, por otros propósitos que los propósitos médicos", de aquí en adelante referidas a "[...] actuaciones imprácticas coordinadas con el diagnóstico, la terapia u otras", o "[...] que no merecen el uso de prácticas designadas a aumentar el número de pacientes, por ejemplo", a través de publicidad deseal, etc. (Etický kódex zdravotníckeho pracouníka, 2004, p. 5752; Etický kódex, 2002, p. 2184). Prácticamente son el ejemplo del respeto por los principios éticos profesionales -el profesionalismo, la legalidad y la prohibición del abuso de poder - los que están íntimamente ligados al problema del aumento de la comercialización de la atención sanitaria. La prueba de la seriedad de este problema es la formulación de los siguientes deberes: "Cuando un médico o un dentista prescribe y recomienda drogas u otros dispositivos médicos, debe manejar los aspectos comerciales, pero sólo con su conocimiento médico, su conciencia y la necesidad del paciente" (Etický kódex, 2002, p. 2184; Lešková Blahová, 2012, p. 110).

Lešková Blahová sostiene que entre otras cosas que el artículo Atención sanitaria profesional y ejercicio de su profesión (como médicos y dentistas) se encuentra típicamente en sintonía con requerimientos bioéticos, por ejemplo: "el trabajador de la salud en relación a los enfermos terminales y a los enfermos agonizantes les alivia el dolor, respeta la dignidad humana y los deseos del paciente según las regulaciones y los medios para aliviar el sufrimiento. La eutanasia y el suicidio asistido son inadmisibles" (Etický kódex zdravotníckeho pracovníka, 2004, p. 5752; Lešková Blahová, 2012, p. 112). Según Lešková Blahová, si uno regresa al preámbulo, su presencia definitivamente aumenta la credibilidad del mismo código de ética, dado que busca exponer los valores morales fundamentales, desde los que es posible derivar principios más específicos y proncipios de ética médica. Los valores universales del Código aconsejan el valor de la vida humana, la salud, la dignidad humana, el sentido de la vida humana y el valor de la familia. Entonces se derivan valores específicos que están asociados con el desempeño de la profesión médica, como la libertad (de pensamiento, conciencia y asociación) y la responsabilidad del médico de protejer y desarrollar valores relacionados a la vida humana, los que concretamente significan respetar el derecho a la vida humana y la salud, la dignidad humana, la integridad física, la identidad biológica y psicológica del ser humano y el valor de la familia (Lekárske odborové združenie, 2012, p. 3; Lešková Blahová, 2012, p. 123).

Rudolf Novotný y Zuzana Novotná argumentan que la Carta de Derechos de los Pacientes de la República Eslovaca en el artículo 8 afirma que el paciente tiene derecho, en un estadio incurable de enfermedad, a alivios y sedantes que correspondan con el conocimiento y posibilidades actuales de la atención sanitaria para los agonizantes. Bajo esta Carta... los enfermos terminales y los agonizantes tienen derecho a un tratamiento humanitario [humane], así como también derecho a estar acompañados en los últimos momentos de su vida por una persona de su elección. Suena como una afirmación muy significativa el hecho de que el paciente tiene el derecho a una muerte digna. En este contexto, es igualmente importante que el paciente tenga derecho al respeto decente de sus pedidos escritos de que no sea resucitado o al rechazo de la aplicación de invertenciones y procedimientos terapéuticos (Novotný - Novotná, 2012, p. 145).

Novotný y Novotná hablan de la necesidad de preservar la dignidad humana en situaciones límite. Los aspectos de pérdida y preservación de la dignidad humana en las etapas finales de enfermedades incurables se relacionan ambos con enfermedades. Esto está vinculado a un grado de independencia (preservar habilidades cognitivas y funciones corporales), controlar los síntomas de la enfermedad (dolor y sintómas somáticos, distrés psicológico: inseguridad, miedo a la muerte). Lo mismo aplica a mantener la dignidad personal (Novotný - Novotná, 2012, p. 146).

Según Novotný y Novotná, es muy importante el esfuerzo por preservar la dignidad social. Esto aplica a mantener la privacidad, accesibilidad y organizaciones de asistencia a la familia y amigos, 
la adecuación de los contactos sociales que apoyan la preservación de la dignidad, no ser llevados a tener que preocuparse por qué clase de problemas sociales traerá su muerte a sus seres queridos. Además, esto se vincula a disponer de sus vidas. Eslovaquia ha reconocido la autonomía del paciente, si con total conciencia y tras ser informado consiente o rechaza el tratamiento (Novotný Novotná, 2012, p. 147; Novotný - Novotná, 2014, pp. 68-70).

\section{Hallazgos empíricos}

En nuestra investigación fueron hechos estudios empíricos entre médicos eslovacos $(\mathrm{N}=68)$ y les fue consultada su opinión con respecto a cuán importante es la dignidad del paciente para los doctores cuando se deciden las prioridades. La opinión positiva definió $\mathrm{N}=21$ (30.9\%) doctores. $\mathrm{N}$ $=34(50 \%)$ tiende a estar de acuerdo con que la dignidad del paciente es una prioridad importante en la decisión. $\mathrm{N}=8(11.8 \%)$ fueron neutrales, y sólo $\mathrm{N}=5(7.4 \%)$ de ellos, estuvieron en desacuerdo con el enunciado previo.

Tabla 1

\begin{tabular}{|l|l|l|}
\hline $\begin{array}{l}\text { Para la mayoría de los doctores la dignidad del paciente } \\
\text { está entre las prioridades importantes al decidir }\end{array}$ & Número & $\mathbf{\%}$ \\
\hline Totalmente de acuerdo con este enunciado & 21 & $30,9 \%$ \\
\hline De acuerdo & 34 & $50,0 \%$ \\
\hline No lo sé & 8 & $11,8 \%$ \\
\hline No estoy de acuerdo & 5 & $7,4 \%$ \\
\hline Totalmente en desacuerdo con el enunciado & 0 & $0,0 \%$ \\
\hline Total & $\mathbf{6 8}$ & $\mathbf{1 0 0} \%$ \\
\hline
\end{tabular}

La siguiente pregunta fue: ¿Qué valores morales entran en su relación con el paciente? Investigamos la importancia de la presencia de estándares morales en el trabajo de doctores en relación al paciente. También en este caso, se ofrece un ránking de valores morales creados sobre la base de las respuestas dadas:

- capacidad de rendir cuentas [accountability] $\mathrm{N}=55$;

- $\quad$ dignidad humana $\mathrm{N}=51$;

- derecho a la vida, $\mathrm{N}=50$;

- humanidad $\mathrm{N}=50$;

- obligación $\mathrm{N}=48$;

- tolerancia $\mathrm{N}=39$;

- justicia $\mathrm{N}=36$

- deber de actuar buscando consecuencias sociales positivas $\mathrm{N}=25$.

Para los doctores estos principios de ética médica son importantes en su trabajo:

- el principio de asistencia 95.5\%;

- el principio de no dañar $86.6 \%$;

- el principio de equidad $62.1 \%$;

- el principio de autonomía $51.5 \%$.

En términos de valores morales en relación a los pacientes los doctores eslovacos prefieren:

- $\quad$ responsabilidad $83.3 \%$;

- derecho a la vida, $75.8 \%$; 
- dignidad humana 77.3\%;

- humanitarismo [humaneness] 75.8\%;

- obligación con los pacientes 72.7\%;

- justicia $54.5 \%$;

- tolerancia $59.1 \%$;

- deber de actuar de acuerdo a consecuencias sociales positivas $39.7 \%$.

También son conscientes de los pros así como también de su crecimiento profesional. Cerca del 42\% de ellos no tiene interés en la educación en ética profesional (seminarios, talleres, bibliografía sobre ética médica) y el 58\% asiste a alguna forma de educación de este tipo.

Por otro lado, los pacientes consideran las siguientes características como muy importantes para los doctores eslovacos:

- $\quad$ profesionalismo $87.6 \%$;

- confiabilidad $83.5 \%$;

- determinación $76.7 \%$;

- humanidad 71.1\%;

- imparcialidad $71.1 \%$;

- $\quad$ amabilidad y cordialidad 67.8\%;

Un 64.4\% corresponde a las expectativas razonables de la conducta profesional. Así el paciente espera de sus doctores un alto grado de erudición, pericia, pero también la responsabilidad por el procedimiento médico y lo relativo a la derterminación y la paz en el temple. En el siguiente puesto está la dignidad (aunque relacionada a la ética médica sea una prioridad). Por lo tanto puede concluirse que los pacientes están dispuestos a tolerar un tipo de doctor o de conducta médica inhumanos, si el profesional tiene un alto grado de pericia. Por ejemplo, son capaces de considerar inapropiados:

- $\quad$ irreverencia $78.4 \%$;

- $\quad$ arrogancia $78.4 \%$;

- impaciencia $72.7 \%$;

- autoritarismo $44.3 \%$

- inconsistencia $43.2 \%$.

Sin embargo, suponen que esto se balancea con un alto nivel de profesionalismo médico de los doctores.

\section{Conclusión}

Como resultado puede concluirse que en cada parte tendría que haber un comité de ética hospitalario, y que así las brechas entre las normas, los principios y los valores éticos podrían ser registrados. Las razones para la creación, el establecimiento y la operación de comités de ética en instalaciones médicas fue un tema complejo en la medicina contemporánea y en la atención sanitaria; un procedimiento de elección más amplio de alternativas en una situación dada; la protección de los trabajadores de la salud, pacientes, y la sociedad; la naturaleza de las decisiones en la práctica médica y de enfermería; el énfasis en la autonomía del paciente y en el paciente mismo; la necesidad de proteger la dignidad, los derechos, la integridad, la identidad y otros intereses legítimos de los pacientes. Sin embargo, vemos que hay muchos problemas en temas concernientes a la dignidad humana dentro de la ética médica en Eslovaquia.

Podemos concluir que la ética de las consecuencias sociales puede ser un punto de partida 
metodológico para la investigación teórica de la dignidad humana así como también para el entrenamiento y la consultoría bioética de la práctica médica en Eslovaquia. Espero que esta sea una herramienta útil en un esfuerzo para mejorar y resolver algunos problemas de la ética médica en Eslovaquia.

\section{www.prometeica.com}

\section{Referencias}

COCHRANE, A. (2010): Undignified Bioethics. In: Bioethics 24(5), pp. 234-241.

DUBIEL-ZIELIŃSKA, P. (2013): "Ethics of Social Consequences" and "Ethics of Development" as Theories Belonging to Stream of Ethics of Act. In: Ethics \& Bioethics (in Central Europe) 3(3-4), pp. 171-188.

ETICKÝ KÓDEX [Code of Ethics]. In: Zákon o povolaní lekára, o Slovenskej lekárskej komore, o povolaní zubného lekára, o Slovenskej komore zubných lekárov a o zmene a doplnení niektorých zákonov č. 219/2002. Príloha k zákonu NR SR č. 219/2002 Z. z., pp. 2184-2185.

ETICKÝ KÓDEX ZDRAVOTNÍCKEHO PRACOVNÍKA [Code of Ethics for Medical Worker]. In: Zákon o poskytovateloch zdravotnej starostlivosti, zdravotníckych pracovníkoch, stavovských organizáciách v zdravotníctve a o zmene a doplnení niektorých zákonov č. 578/2004. Príloha č. 4. k zákonu NR SR č. 578/2004 Z. z., pp. 5752-5753.

FOSTER, C. (2015): Human dignity in bioethics and law. In: Journal of Medical Ethics 41(12), p. 935 .

GLUCHMAN, V. (2012): Ethics of Social Consequences - Methodology of Bioethics Education. In: Ethics \& Bioethics (in Central Europe) 2(1-2), pp. 16-27.

GLUCHMANOVÁ, M. (2009): Principles of ethics of social consequences and teaching of bioethics. In: V. Gluchman (ed.): Bioethics in Central Europe. Prešov: FF PU, pp. 243-250.

HARNACKE, C. (2016): The Ashley Treatment: Improving Quality of Life or Infringing Dignity and Rights? In: Bioethics 30(3), pp. 141-150.

KALAJTZIDIS, J. (2013): Ethics of Social Consequences as a Contemporary Consequentialist Theory. In: Ethics \& Bioethics (in Central Europe) 3(3-4), pp. 159-171.

KOMENSKÁ, K. (2012a): Reflexia princípov medicínskej etiky v profesijných kódexoch na Slovensku. In: V. Gluchman a kol.: Profesijná etika (Analýza stavu profesijnej etiky na Slovensku). Prešov: FF PU, pp. 79-99.

KOMENSKÁ, K. (2012b): Respect for autonomy and human dignity in codes of conduct of health care professionals (in Slovakia). In: Ethics \& Bioethics (in Central Europe) 2(3-4), pp. 192-200.

KOMENSKÁ, K. (2011): The analysis of ethical codes in health care professions in Slovakia (professions of physicians, nurses and midwives). In: Ethics \& Bioethics (in Central Europe) 1(12), pp. 25-32.

LEKÁRSKE ODBOROVÉ ZRUŽENIE (2012): Etický kódex [Code of ethics]. Available at: http://www.loz.sk/system/files/eticky_kodex_LOZ_25_2_2012_final_cisty_2.pdf [Retrieved: 21 October 2016]. 
LEŠKOVÁ BLAHOVÁ, A. (2012): Kodifikovaná podoba lekárskej etiky na Slovensku. In: V. Gluchman a kol.: Profesijná etika (Analýza stavu profesijnej etiky na Slovensku). Prešov: FF PU, pp. 101-130.

LEŠKOVÁ BLAHOVÁ, A. (2009): Possibilities of ethics of social consequences application in bioethics contexts. In: V. Gluchman (ed.): Bioethics in Central Europe. Prešov: FF PU, pp. 87-99.

MACKLIN, R. (2003): Dignity is a Useless Concept. In: British Medical Journal vol. 327, pp. 14191420.

MISSERI, L. E. (2015): Consequentialism, humankind and dignity: From Renaissance moral philosophy to Gluchman's ethics of social consequences. In: Ethics \& Bioethics (in Central Europe) 5(3-4), pp. 197-203.

NOVOTNÝ, R. - NOVOTNÁ, Z. (2014): Bioethical Principles in Clinical Medicine. In: Ethics \& Bioethics (in Central Europe) 4(1-2), pp. 67-72.

NOVOTNÝ, R. - NOVOTNÁ, Z. (2012): Profesijná etika v odbore vnútorné lekárstvo v Slovenskej republike. In: V. Gluchman a kol.: Profesijná etika (Analýza stavu profesijnej etiky na Slovensku). Prešov: FF PU, pp. 131-159.

PALACIOS-GONZÁLES, C. (2015): Human dignity and the creation of human-nonhuman chimeras. In: Medicine, Health Care and Philosophy 18(4), pp. 487-499.

PILKINGTON, B. C. (2016): Dignity, Health, and Membership: Who counts as One of Us? In: Journal of Medicine and Philosophy, 41(2), pp.115-129.

PINKER, S. (2008): The Stupidity of Dignity. In: The New Republic 238(9), pp. 28-31.

PIRHONEN, J. (2015): Dignity and the capabilities approach in long-term care for older people. In: Nursing Philosophy 16(1), pp. 29-39.

SACHDEV, K. N. (2015): Normative analysis of human dignity among professionals. In: Ethics \& Bioethics (in Central Europe) 5(3-4), pp. 205-210.

SCHÜKLENK, U. - PACHOLCZYK, A. (2010): Dignity's Wooly Uplift. In: Bioethics 24(2), p. ii.

UNESCO (2000): The Universal Declaration on the Human Genome and Human Rights: From Theory to Practice. Paris: UNESCO.

UNESCO (2006): Universal Declaration on Bioethics and Human Rights. Paris: UNESCO.

ZÁKON č. 311/2002 Z. z. o povolaní sestry, o povolaní pôrodnej asistentky, o Slovenskej komore sestier a pôrodných asistentiek a o zmene a doplnení zákona Slovenskej národnej rady č. 14/1992 Zb. o Slovenskej komore stredných zdravotníckych pracovníkov a o Slovenskej komore zubných technikov.

ZÁKON č. 578/2004 Z. z. o poskytovatel'och zdravotnej starostlivosti, zdravotníckych pracovníkoch, stavovských organizáciách v zdravotníctve a o zmene a doplnení niektorých zákonov. 